\title{
Image Segmentation Using Normalized Cuts and Efficient Graph-Based Segmentation
}

\author{
Narjes Doggaz and Imene Ferjani \\ URPAH, Computer Science Department, Faculty of Sciences of Tunis, Tunisia \\ narjes.doggaz@fst.rnu.tn, \\ imene.fer@gmail.com
}

\begin{abstract}
In this paper we propose an hybrid segmentation algorithm which incorporates the advantages of the efficient graph based segmentation and normalized cuts partitioning algorithm. The proposed method requires low computational complexity and is therefore suitable for realtime image segmentation processing. Moreover, it provides effective and robust segmentation. For that, our method consists first, at segmenting the input image by the "Efficient Graph-Based" segmentation. The segmented regions are then represented by a graph structure. As a final step, the normalized cuts partitioning algorithm is applied to the resulting graph in order to remove non-significant regions. In the proposed method, the main computational cost is the efficient graph based segmentation cost since the computational cost of partitioning regions using the Ncut method is negligibly small. The efficiency of the proposed method is demonstrated through a large number of experiments using different natural scene images.
\end{abstract}

Keywords: Image Segmentation, Normalized Cuts, Efficient graph-based, Region adjacency graph.

\section{Introduction}

Segmentation is defined as the process of partitioning an image into disjoint and homogeneous regions. It is an important step in image analysis. Besides, it is one of the most difficult tasks in image processing, and determines the quality of the final results of the computer vision applications.In the literature, several segmentation methods are defined. They can be classified into three major categories,i.e.,feature based techniques, physics based techniques and image-based techniques. Feature-based methods, such as clustering [4], intend to classify pixels to different groups in a pre-defined color space. These methods gives good results in some cases [19] but their drawbacks are neglecting the spatial information of pixels and the difficulty met in adjusting the number of classes to image regions. The objective of physics based segmentation is to divide an image of a scene in regions that are meaningful in terms of the objects constituting that scene by forming an interpretation of the image based on illumination, material optics and geometry 15. These methods alleged robustness to highlights, 
shades and shadowing, but they are not applicable for real images. Image-based techniques are also referred to as region-based when they are based on region entities. Region growing methods 20 are extensively used for this purpose. In such methods regions are iteratively grown by comparing all unallocated neighboring pixels to the regions. These methods can produce an oversegmentation which can be avoided by applying some merging algorithms. Also, graph theoretical methods are considered as region based methods and they have proved their robustness in many image analysis applications, this is why they will be detailed separately.

This paper is organized as follows: in section 2 we explicate the principle of graph theoretical segmentation methods and we focus on efficient graph-based segmentation and normalized cut partitioning algorithm. In section 3, we present and detail our approach that combines the two above methods. In section 4, we present and discuss the results of our approach as well as its computational complexity and we conclude in section 5 .

\section{Graph Theoretical Segmentation Methods}

Graph-based approaches can be regarded as image perceptual grouping and organization methods based on the fusion of the feature and spatial information. The common theme underlying these approaches is the formation of a weighted graph, where each vertex corresponds to an image pixel or a region. The weight of each edge connecting two pixels or two regions represents the likelihood that they belong to the same segment. A graph is partitioned into multiple components that minimize some cost function of the vertices in the components and/or the boundaries between those components. Several graph theoreticalbased methods have been developed for image segmentation [24, 23]. Some are region growing methods that join components as a function of the attributes of nodes and edges and others are splitting methods that partition a graph by removing superfluous edges. The efficient graph based method proposed by Pedro et al [10] can be classified as a region growing method. It is highly efficient and cost effective way to perform image segmentation. In splitting methods, the normalized cuts algorithm [17] is extensively used in image segmentation due to its efficiency according to traditional minimum cuts algorithms. However, segmentation based on normalized cuts needs high computational time. Several solutions were presented to solve this problem. Some approaches propose to apply the Ncuts partitioning algorithm to the graph representing a segmented image. By this way there is a great reduction of the computational time since the number of regions obtained by the first segmentation is much smaller than the number of image pixels. But the quality of final segmentation differs from one solution to another. In the work of Makrogiannis et al [13] the image is segmented by applying the watershed algorithm. A graph structure is then applied to represent the relationship between these regions, this resulting graph will be partitioned 
using Ncuts algorithm. In this method the oversegmentation produced by the watersheds causes a degradation in the region grouping algorithm. To solve this problem Tao et al 18 proposed a similar method which uses mean-shift instead of watershed segmentation, and to avoid inappropriate partitioning, they represent a region with multiple child nodes. In both approaches there is a great reduction of the Ncut cost but the segmentation obtained can not be integrated in real time applications without the need to use a parallel environment. Also, performance in terms of extracting objects from the image is still insufficient. In our work, we use two graph theoretical based methods which are efficient graph based segmentation [10] and the Ncuts partitioning algorithm [17] to provide robust real time segmentation.

\subsection{Normalized Cuts Segmentation}

A graph-partitioning method attempts to organize nodes into groups such that the intra-group similarity is high and the inter-group similarity is low. Given a graph $\mathrm{G}=(\mathrm{V}, \mathrm{E}, \mathrm{W})$, where $\mathrm{V}$ is the set of nodes, and $\mathrm{E}$ is the set of edges connecting the nodes, a pair of nodes $u$ and $v$ is connected by an edge and is weighted by $w(u, v)=w(v, u) \geq 0 . w(u, v)$ measures the dissimilarity between $u$ and $v . \mathrm{W}$ is an edge affinity matrix with $w(u, v)$ as its $(u, v)^{t h}$ element. The graph can be partitioned into two disjoint sets $\mathrm{A}$ and $\mathrm{B}=\mathrm{V}-\mathrm{A}$ by removing the edges connecting the two parts. The degree of dissimilarity between the two sets can be computed as a total weight of the removed edges. In graph theoretic language, it is called the cut[17].

$$
\operatorname{cut}(A, B)=\sum_{u \in \operatorname{Aet} v \in B} w(u, v)
$$

The problem of finding the minimum cut has been well studied. However, the minimum cut criterion favors grouping small sets of isolated nodes in the graph because the cut defined in equation 1 does not contain any intra-group information. In other words, the minimum cut usually yields overclustered results when it is recursively applied. To avoid this unnatural bias of partitioning out small sets of points, Shi and Malik propose a new measure of dissociation between two groups called Normalized cut(Ncut) [17].

$$
N \operatorname{cut}(A, B)=\frac{\operatorname{cut}(A, B)}{\operatorname{assoc}(A, V)}+\frac{\operatorname{cut}(A, B)}{\operatorname{assoc}(B, V)}
$$

where $\operatorname{assoc}(A, V)=\sum_{u \in A, t \in V} w(u, t)$ denotes the total connection from nodes in A to all nodes in the graph, and $\operatorname{assoc}(B, V)$ is similarly defined. Unlike the cut criterion that has a bias in favor of cutting small sets of nodes, the Ncut criterion is unbiased [17. 


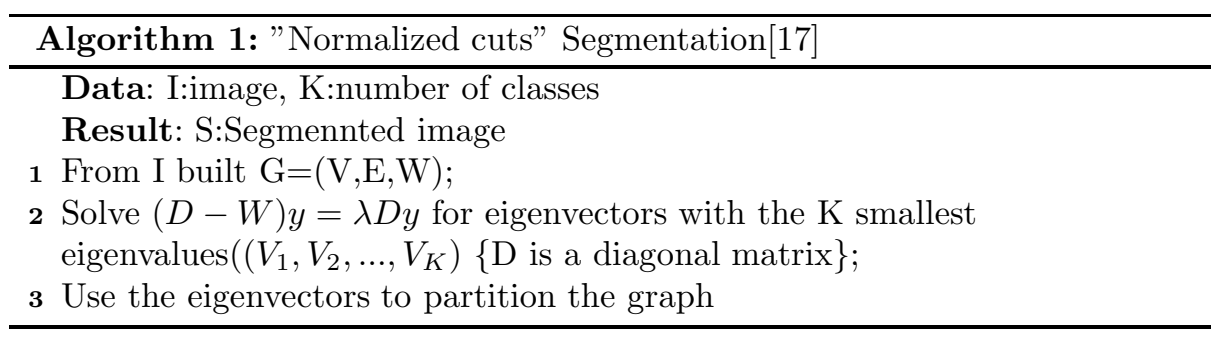

\subsection{Efficient Graph-Based Segmentation}

The graph based image segmentation is based on selecting edges from a graph, where each pixel corresponds to a node in the graph 10 . Weight on each edge measures the dissimilarity between pixels. The segmentation algorithm defines the boundaries between regions by comparing two quantities:Intensity differences across the boundary and Intensity difference between neighboring pixels within each region. This is useful knowing that the intensity differences across the boundary are important if they are large relative to the intensity differences inside at least one of the regions. This results in a method that obeys certain non-obvious global properties. Let the internal difference of a component $\mathrm{C}$ in an image be [10]:

$$
\operatorname{Int}(C)=\max _{e \in M S T(C, E)} w(e)
$$

Where $w(e)$ is the largest weight in the Minimum Spanning Tree of the component. Let the difference between two components $C_{1}$ and $C_{2}$ be the minimum weight edge connecting the two components. That is [10,

$$
\operatorname{Dif}\left(C_{1}, C_{2}\right)=\min _{v_{i} \in C_{1}, v_{j} \in C_{2},\left(v_{i}, v_{j}\right) \in E} w\left(\left(v_{i}, v_{j}\right)\right)
$$

The boundary between a pair of components is determined by checking if the difference between the components, $\operatorname{Dif}\left(C_{1}, C_{2}\right)$, is large relative to the internal difference within at least one of the components, $\operatorname{Int}\left(C_{1}\right)$ and $\operatorname{Int}\left(C_{2}\right)$. A threshold function is used to determine the degree to which the difference between components must be larger than minimum internal difference.Let the predicate $\mathrm{D}$ be [10]:

$$
D\left(C_{1}, C_{2}\right)=\left\{\begin{array}{l}
\text { true, } \text { if } \operatorname{Dif}\left(C_{1}, C_{2}\right)>M \operatorname{Int}\left(C_{1}, C_{2}\right) ; \\
\text { false, otherwise. }
\end{array}\right.
$$

Where the minimum internal difference MInt is defined as [10]:

$$
M \operatorname{Int}\left(C_{1}, C_{2}\right)=\min \left(\operatorname{Int}\left(C_{1}\right)+\tau\left(C_{1}\right), \operatorname{Int}\left(C_{2}\right)+\tau\left(C_{2}\right)\right)
$$

with

$$
\tau(C)=K /|C|
$$

$|C|$ is the size of the component $C$, and $K$ is a constant parameter. 


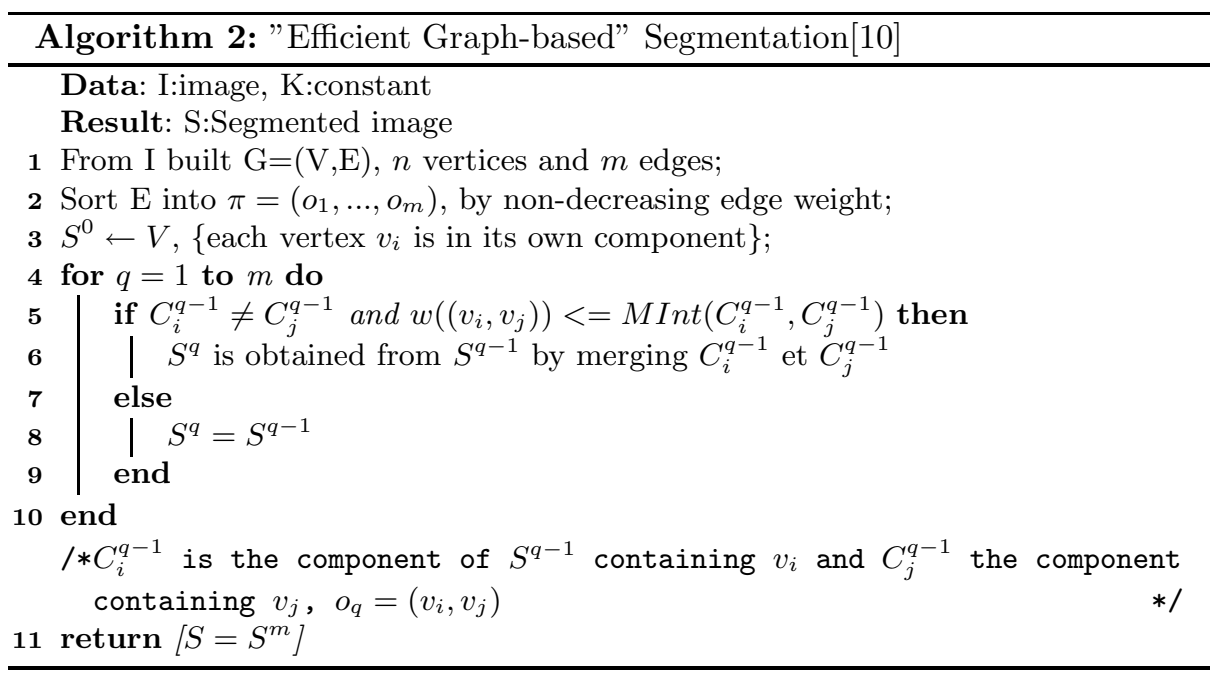

\section{Our Approach}

The outline of our approach can be defined as following. First, an image is segmented into separated regions using the efficient graph based algorithm. Second, the graph representation of these regions is constructed, and the dissimilarity measure between the regions is defined. Finally, a graph-partitioning algorithm based on the Ncuts is employed to form the final segmentation map (fig. 1).

The most important part in our approach is the construction of the region adjacency graph (RAG) and the calculation of the dissimilarity measure between the regions.

The regions obtained by the "Graph-Based" segmentation can be represented by a weighted region adjacency graph $\mathrm{G}=(\mathrm{V}, \mathrm{E}, \mathrm{W})$. The weights on the edges of RAG based on the similarity between two regions play a decisive role in determining the overall performance of our image segmentation process. To define the measure of dissimilarity between neighboring regions, we propose the use of the mean intensity of regions and their compactness [2]. The compactness between two regions is defined as the degree of their adjacency(how much they exhibit adjacency of their constituent parts) since they are considered adjacent when they have at least one adjacent pixel. In our approach, we used the compactness criteria introduced by Adamek which is defined by [2]:

$$
C_{i j}=1-\frac{F_{i, j}}{\min \left\{L_{i}, L_{j}\right\}}
$$

Where $L_{i}$ and $L_{j}$ are the perimeters of regions $\mathrm{i}$ and $\mathrm{j}$ and $F_{i, j}$ is the length of their common border. $C_{i j}$ gives a value between 0 and 1 . When $C_{i j}=0$ this means that one of regions ( $\mathrm{i}$ or $\mathrm{j}$ ) is totally included in the other region. When $C_{i j}=1$ this means that the regions $\mathrm{i}$ and $\mathrm{j}$ are not adjacent. 


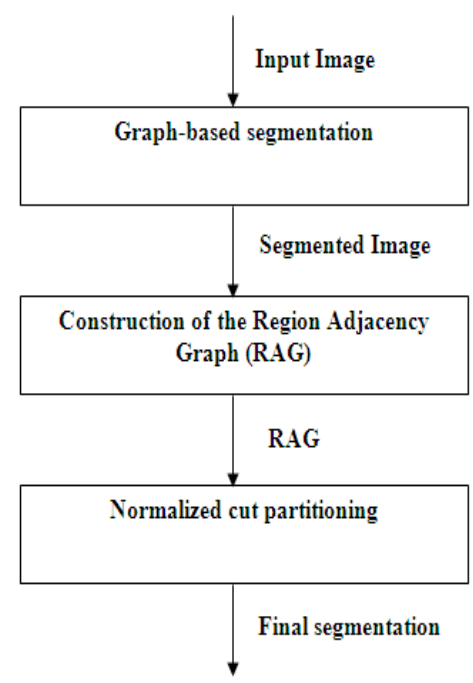

Fig. 1. The outline of our approach

We can now propose our definition of the weight $w(i, j)$ between regions $\mathrm{i}$ and $\mathrm{j}$ by:

$$
w(i, j)=\exp \left(\frac{-\left\|I_{i}-I_{j}\right\|^{2}}{\sigma_{I}^{2}}\right) * \begin{cases}\exp -\left\|C_{i j}\right\|, & \text { if } \mathrm{i} \text { et } \mathrm{j} \text { are adjacents } \\ 0, & \text { otherwise }\end{cases}
$$

Where $I_{i}$ is the mean intensity of region $i$ and the constant $\sigma_{I}$ is the same used in the Ncuts segmentation algorithm. The first factor of the above equation is the mean intensity difference between two adjacent regions $i$ and $j$. The second factor is used to favorise the creation of compact and simple regions.

Once, the region adjacency graph constructed, we apply the normalized cuts algorithm to partition the graph and to group similar regions. The advantages of using hybrid segmentation instead of using separately efficient graph based and normalized cuts segmentation are twofold:

1) It improves segmentation performance. Graph based image-segmentation is a fast and efficient method of generating a set of segments from an image. It not only considers local pixel-based features, but also looks at global similarities within the image but its drawback is that the number of regions generated is relatively high.

2)We can use the Ncuts algorithm as a post processing step without increasing the computational time of the segmentation process because partitioning regions instead of pixels will offer considerable reduction of computational complexity, since the number of image regions is much smaller than that of the pixels. Thus, the size of the weight matrix and, subsequently, the complexity of the graph structure employed for image representation are significantly reduced. Moreover, 
applying Ncuts partitioning to regions instead of pixels is more accurate and easy since the edge's weight between tow regions is larger than that between two pixels.

\section{Experimental Results}

We have applied the proposed algorithm on the Berkeley Segmentation Dataset [3. where the images are $321 \times 481$ pixels. We present in fig. 3. fig. 4 and fig. 5] the results of some of our experiments. For each image, the result of the "GraphBased" algorithm [1, the Ncuts algorithm implemented by Cour et al [7], our segmentation approach and the human segmentation are given. Hence, we can compare visually and intuitively the result of each method of segmentation with the ground truth segmentation.

In the implementation of the "Graph-Based" segmentation there is a pretreatment step to reduce noise. This step needs a smoothness factor $\sigma$. Also, there is a post-treatment step that eliminates small regions after applying the segmentation algorithm. The parameter minsize is introduced to define the smallest size that a region can have. The parameters of "Graph-Based" segmentation algorithm are $(K, \sigma$, minsize $)=(1000,1,100)$. We remark that the "Efficient graph-based" segmentation extracts the most important objects in the image but it produces a large number of regions(fig. 3r, fig. 4 c and fig. 5r). We remark clearly that the number of these regions in our approach has been decreased in a way that preserves the global structure of the image segmentation. To more illustrate our results let focus in the image of fig. 2 which contains two flowers.
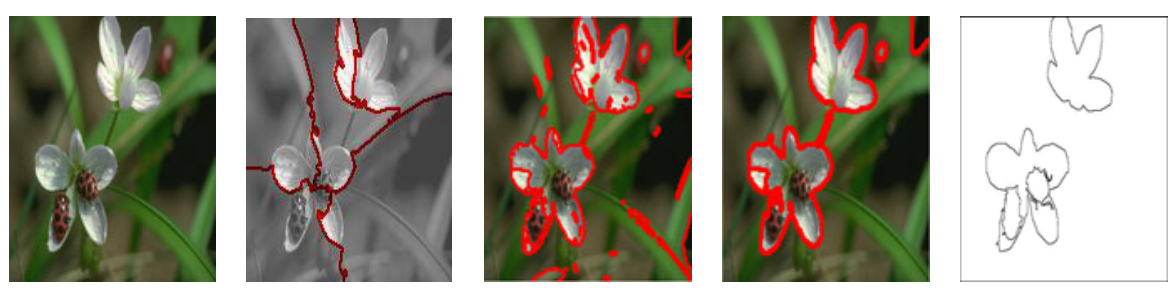

Fig. 2. (a)The original test image. (b)Normalized cuts segmentation. (c)Efficient graph based segmentation. (d)Our proposed method. (e)Human segmentation.

In this example, the segmentation produced by efficient graph based method contains 43 regions. We remark, when we apply our algorithm with setting the number of classes to 6 , we obtain a segmentation that preserve the most important objects in the image wich are the two flowers. The regions that belong to the background are also formed into a single region. However, when the Ncut method is directly applied to the image pixels with the same number of classes, the image is partitioned into six regions where each region includes one part of each flower. Our segmentation method distinguishes more objects from the background. By 
comparing our segmentation results to the ground truth segmentations it is clear that our results are the most similar to the human segmentation. Regions extracted by our method are more meaningful in most of images.
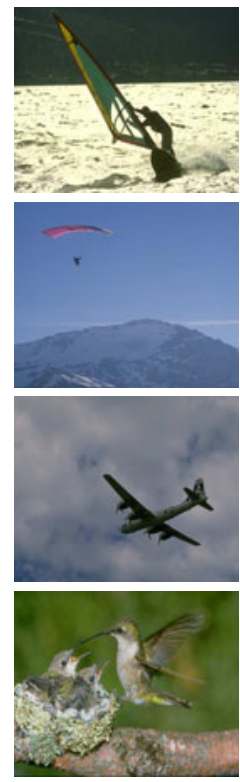

(a)
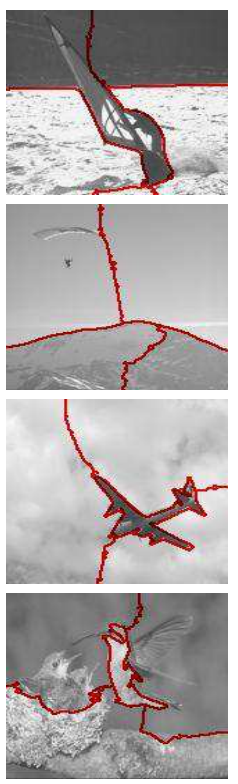

(b)
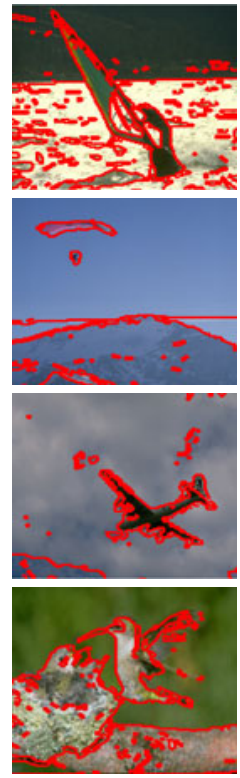

(c)

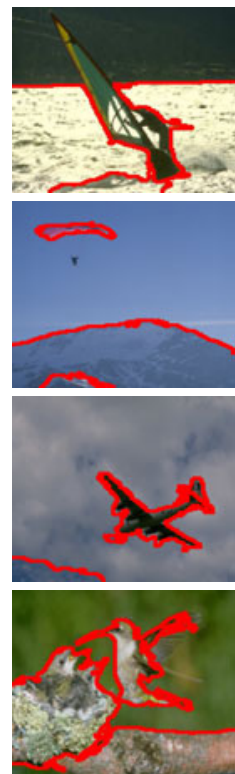

(d)

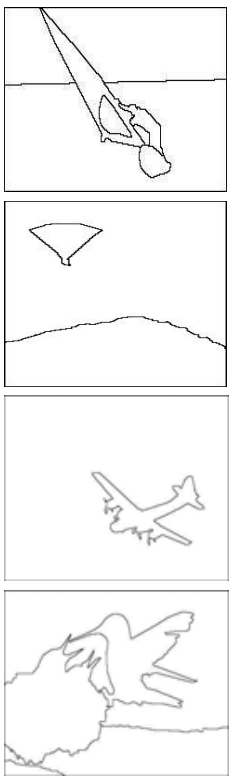

(e)

Fig. 3. Test results of different images with partitioning class $C=4$. (a) The original test images. (b)Normalized cuts segmentation. (c)Efficient graph based segmentation. (d)Our proposed method. (e)Human segmentation.

We evaluate, also, and compare the computational cost of our method with those of the Graph-Based and Ncut ones. For that, we use a PC equipped with a 1.6-Ghz and 1-GB memory. We consider only the first four images from up to bottom in each of fig. 3 f fig. 4 and fig. 5 . We notice that the computational cost of our approach is near to the "Graph-Based" method which takes 700-800 ms to process an image. This means that the cost of partitioning the region nodes using the Ncut method is negligibly small. However, the normalized cuts segmentation takes $9-19$ s to segment an image [1.

To evaluate objectively the segmentation results given by our approach we use four different measures that are:

Probabilistic Rand Index (PRI) [21]: PRI counts the number of pixel pairs whose labels are consistent between the segmentation and the ground truth. Variation of Information (VoI) [16]: VoI measures the amount of randomness in one segmentation that cannot be explained by the other. 

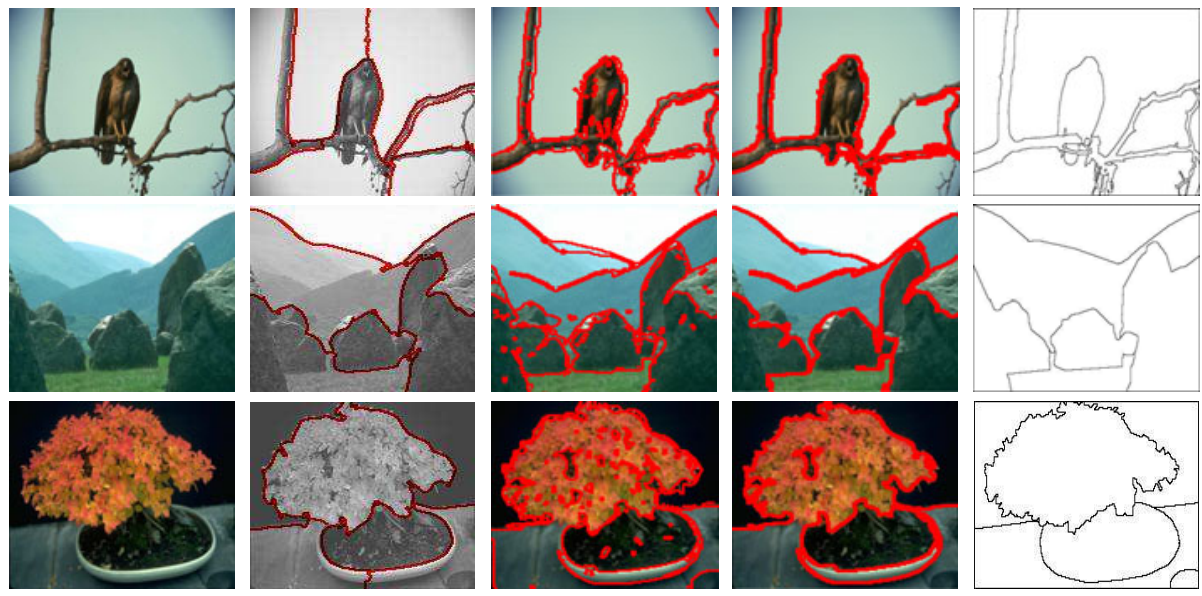

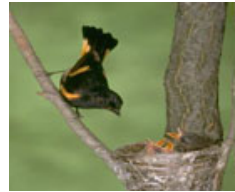

(a)

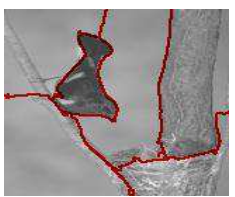

(b)

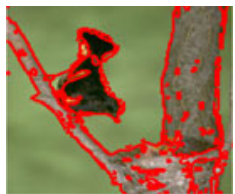

(c)

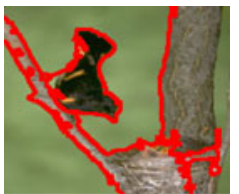

(d)

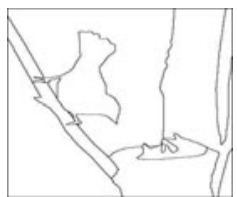

(e)

Fig. 4. Test results of different images with partitioning class $\mathrm{C}=6$. (a) The original test images. (b)Normalized cuts segmentation. (c)Efficient graph based segmentation. (d)Our proposed method. (e)Human segmentation.

Table 1. Comparaison of the computational cost between "Graph-Based", Ncuts and Our method

\begin{tabular}{|c|c|c|c|c|}
\hline & & $\begin{array}{l}\text { "Graph-Based" time } \\
\text { (s) }\end{array}$ & Ncuts time(s) & Our method time(s) \\
\hline & 1 & 0.777 & 13.297 & 0.940 \\
\hline & 2 & 0.783 & 10.023 & 0.8921 \\
\hline $\mathrm{C}=4$ & 3 & 0.782 & 9.917 & 0.876 \\
\hline & 4 & 0.779 & 15.057 & 0.919 \\
\hline & 1 & 0.775 & 14.584 & 0.898 \\
\hline $\mathrm{C}=\mathrm{G}$ & 2 & 0.814 & 14.860 & 0.945 \\
\hline$c=0$ & 3 & 0.764 & 14.747 & 0.901 \\
\hline & 4 & 0.832 & 15.057 & 0.984 \\
\hline & 1 & 0.770 & 15.912 & 0.948 \\
\hline $\mathrm{C}-8$ & 2 & 0.784 & 19.563 & 0.957 \\
\hline $\mathrm{C}=\mathrm{\gamma}$ & 3 & 0.821 & 14.747 & 0.986 \\
\hline & 4 & 0.765 & 13.082 & 0.921 \\
\hline
\end{tabular}




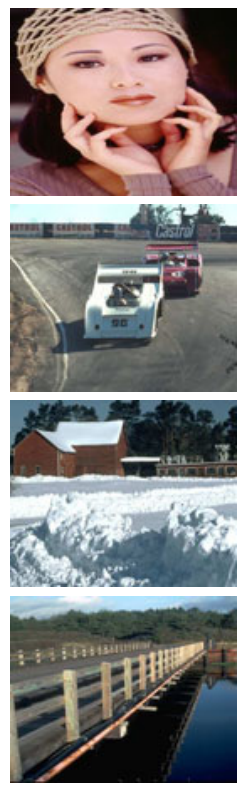

(a)

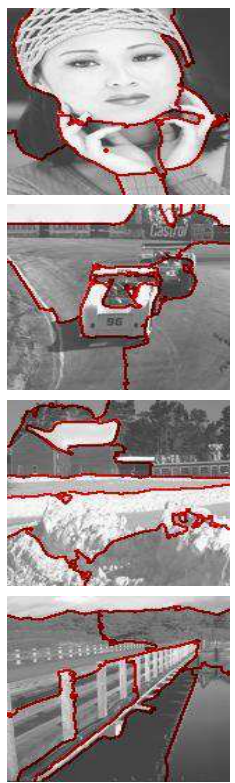

(b)

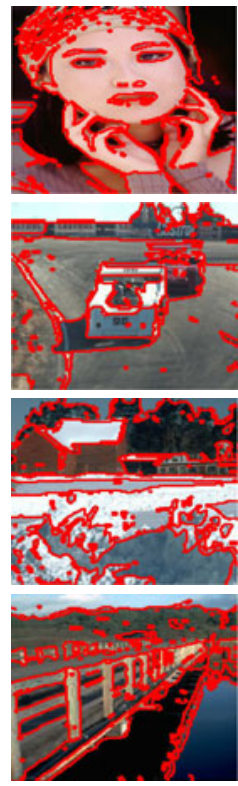

(c)

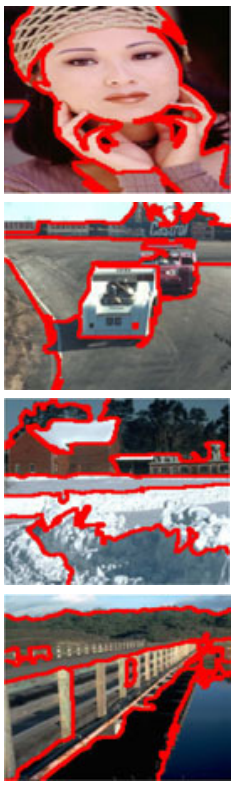

(d)
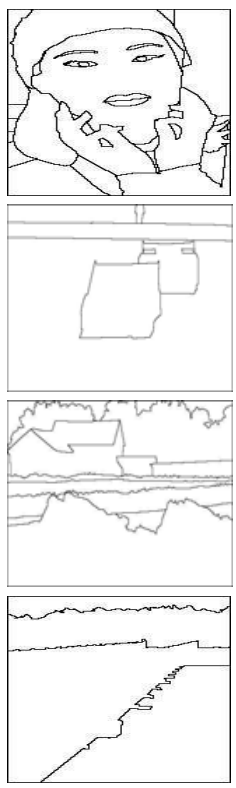

(e)

Fig. 5. Test results of different images with partitioning class $\mathrm{C}=8$. (a) The original test images. (b)Normalized cuts segmentation. (c)Efficient graph based segmentation. (d)Our proposed method. (e)Human segmentation.

Table 2. Quantitative comparison of our algorithm with other segmentation methods over the Berkeley database. The best three results are highlighted in colors: Red, Green, and Blue in descending order.

\begin{tabular}{|c|c|c|c|c|}
\hline Method Score & PRI & VOI & GCE & $\mathrm{BDE}$ \\
\hline Mshift [5] & 0.7958 & 1.9120 & 0.1888 & 14.41 \\
\hline Ncuts[17] & 0.7242 & \begin{tabular}{|l|}
2.9061 \\
\end{tabular} & 0.2232 & 17.15 \\
\hline Jseg $[8$ & 0.7756 & 2.3217 & 0.1989 & 14.40 \\
\hline SpectClust [6] & 0.7357 & 2.6336 & 0.2469 & 15.40 \\
\hline GraphBased 10] & 0.7139 & 3.3949 & 0.1746 & 16.67 \\
\hline Mscuts [25] & 0.7559 & 2.4701 & 0.1925 & 15.10 \\
\hline ROI-Seg[9] & 0.7599 & 2.0072 & 0.1846 & 22.45 \\
\hline NormTree 22 & 0.7521 & 2.4954 & 0.2373 & 16.30 \\
\hline Our method & 0.6902 & 1.9028 & 0.1580 & 19.7984 \\
\hline
\end{tabular}

Global Consistency Error (GCE) [14]: GCE measures the extent to which one segmentation can be viewed as a refinement of the other.

Boundary Displacement Error (BDE) [11]: BDE measures the average displacement error of the boundary pixels between two segmented images. 
We notice that, more is greater the PRI measure, better is the segmentation. In the other hand, more are smaller the VoI, GCE and BDE measures, better is the segmentation. The choice of these four measures where motivated by the fact that those measures where used to evaluate some image segmentation algorithms. Thus, we can compare objectively our segmentation method with those given in 12 .

We report in table 2, the average scores over the Berkeley database for eight other segmentation methods: Mean Shift(MShift) [5], "Normalized cuts" (NCut) [17], Graph-based Segmentation [10, Spectral clustering((Spect-Clust) [6], multi-scale normalized cut(Mscuts) 25], MSER-based segmentation (ROISeg) 9], normalized partitioning tree (NormTree) [22 and the JSEG algorithm (Jseg) 8]. We set the number of regions in our method to 6 for all the database images. We remark that our method achieves the best performance for VoI and GCE measures. This comparison proves that our segmentation method have less errors in terms of variation of information with respect to the ground truth.

\section{Conclusion}

In this work, we proposed an hybrid segmentation based on efficient graph based segmentation and normalized cuts partitioning algorithm. The proposed method segment an image with the efficient graph based method, computes the region adjacency graph from the segmented image and finally partition the graph to have the final segmentation. This approach has shown good result with a low computational time.

\section{References}

1. Graph based image segmentation tutorial (November 21, 2007), http://www.cis.upenn.edu/ jshi/graphtutorial/

2. Adamek, T., Connor, E., Murphy, N.: Region-based segmentation of images using syntactic visual features. In: 6th International Workshop on Image Analysis for Multimedia Interactive Services (April 2005)

3. Berkeley: Berkeley segmentation and boundary detection benchmark and dataset (2003), http://www.cs.berkeley.edu/projects/vision/grouping/segbench

4. Chen, T.W., Chen, Y.L., Chien, S.Y.: Fast image segmentation based on k-means clustering with histograms in hsv color space. In: Multimedia Signal Processing, pp. 322-325 (2008)

5. Comaniciu, D., Meer, P.: Mean shift: a robust approach toward feature space analysis. IEEE Transactions on Pattern Analysis and Machine Intelligence 24, 603-619 (2002)

6. Cour, T., Benezit, F., Shi, J.: Spectral segmentation with multiscale graph decomposition. In: Computer Vision and Pattern Recognition, CVPR (2005)

7. Cour, T., Yu, S., Shi, J.: Normalized cuts matlab code, http://www.cis.upenn.edu/ jshi/software

8. Deng, Y., Manjunath, B.S.: Unsupervised segmentation of color-texture regions in images and video. Pattern Analysis and Machine Intelligence 35, 800-810 (2001) 
9. Donoser, M., Bischof, H.: Roi-seg: Unsupervised color segmentation by combining differently focused sub results. In: Computer Vision and Pattern Recognition (CVPR) (2007)

10. Felzenszwalb, P., Huttenlocher, P.: Efficient graph-based image segmentation. International Journal of Computer Vision 59, 167-181 (2004)

11. Freixenet, J., Muñoz, X., Raba, D., Martí, J., Cufí, X.: Yet another survey on image segmentation: Region and boundary information integration. In: Heyden, A., Sparr, G., Nielsen, M., Johansen, P. (eds.) ECCV 2002. LNCS, vol. 2352, pp. 408-422. Springer, Heidelberg (2002)

12. Hoon, T., Mu, K., Uk Lee, S.: Learning full pairwise affinities for spectral segmentation. In: Computer Vision and Pattern Recognition (CVPR), pp. 2101-2108 (2010)

13. Makrogiannis, S., Economou, G., Fotopoulos, S.: A region dissimilarity relation that combines feature-space and spatial information for color image segmentation. IEEE Transactions Systems, Man, Cybernetics Part B 35, 44-53 (2005)

14. Martin, D., Fowlkes, C., Malik, J.: A database of human segmented natural images and its application to evaluating segmentation algorithms and measuring ecological statistics. Technical report, EECS Department. University of California, Berkeley, Janvier (2001)

15. Maxwell, A., Shafer, A.: Physics-based segmentation of complex objects using multiple hypotheses of image formation. Computer Vision And Image Understanding 65, 269-295 (1997)

16. Meila, M.: Comparing clusterings by the variation of information. Journal of Multivariate Analysis, 173-187 (2003)

17. Shi, J., Malik, J.: Normalized cuts and image segmentation. IEEE Transaction on Pattern Analysis and Maching Intelligence 22, 888-905 (2000)

18. Tao, W., Jin, H., Zhang, Y.: Color image segmentation based on mean shift and normalized cuts. IEEE Transactions on, Systems and Cybernetics-Part B 37, 1382 1388 (2007)

19. Tatiraju, S., Mehta, A.: Image segmentation using k-means clustering, em and normalized cuts. Technical report

20. Thiran, J., Warscotte, V., Macq, B.: A queue-based region growing algorithm for accurate segmentation of multi-dimensional digital images. Signal Processing 60, $1-10$ (1997)

21. Unnikrishnan, R., Pantofaru, C., Hebert, M.: Toward objective evaluation of image segmentation algorithms. Pattern Analysis and Machine Intelligence 29, 929-944 (2007)

22. Wang, J., Jia, Y.: Normalized tree partitioning for image segmentation. In: Computer Vision and Pattern Recognition, CVPR (2008)

23. Weiss, Y.: Segmentation using eigenvectors: A unifying view. In: Seventh International Conference on Computer Vision (ICCV 1999), vol. 2, p. 975 (1999)

24. Wu, Z., Leahy, R.: An optimal graph theoretic approach to data clustering: Theory and its application to image segmentation. IEEE Transactions on Pattern Analysis and Machine Intelligence 15, 1101-1113 (1993)

25. Yu, S., Shi, J.: Multiclass spectral clustering. In: International Conference on Computer Vision (ICCV), pp. 313-319 (2003) 